complet et nous savons bien que e'est peu d'aroir seulement indiqué ces problèmes. Nous savons aussi que toute une série de ces problèmes sont d'une grande difficulté scientifigue. Il ne peut done s'agir que d'un programme à larges vues. Malgré cela, tout essai qui s'occupera sérieusement de résoudre un de ces nombreux problèmes au point de vue de la pratigue de la fromagerie, sera le bienvenu.

(A suivre.)

\title{
ÉTUDE COMPARATIVE DES PRINCIPALES CONSTANTES UTILISÉES POUR LA DÉTERMINATION DU MOUILLAGE DU LAIT (1)
}

\author{
par J. CERE
}

Ingénieur de l'Institut agricole et diplômé de l'Ecole de Laiterie de la Faculté des Sciences de Nancy, Ancien assistant de l'Etablissement fédéral d'Industrie laitière et de Bactériologie du Liebefeld, Berne.

INTRODUCTION.

Définition. - En chimie laitière, on appelle "constantes" des formules chiffrées résultant d'analyses multiples, qu'on a eherché à rendre constantes, c'est-à-dire stables.

Or, nous dit DUCLaUX : " Rien n'est moins constant que les constantes du lait. " Cet aphorisme n'est nullement une boutade, car ces constantes n'ont en effet pas toujours la même valeur. Elles varient selon le procédé qui leur a donné naissance. C'est pourquoi DucLaux a pu dire également : "Il n'y a pas un lait, il y a des laits. "

Il est évident que la composition chimique du lait varie par suite de causes nombreuses. Certains éléments constitutifs de ce liquide s'y rencontrent en quantité dont les proportions peuvent osciller entre 1 et $50 \%$. Cet état de choses rend la détermination des fraudes délicate et aléatoire, si l'on s'en tient à l'analyse quantitative des éléments qui entrent dans la composition du lait.

Pour obvier à ces inconvénients, les chimistes ont eu recours à d'autres procédés physiques ou chimiques indépendants des variations quantitatives. Ces procédés, traduits en formules chiffrées, s'appellent, comme nous l'avons dit : "Constantes du lait ".

Suivant leur origine, nous les elassons en deux catégories :

1. Les constantes physiques.

2. Les constantes chimiques.

Malheureusement ces constantes, fort nombreuses, sont d'inégale valeur et les meilleures, peut-être, sont ou trop peu connues, ou mal comprises. Leur application maladroite provoque souvent des résultats contradictoires qui justifient les reproches qu'on leur fait. Aussi comprenons-

(1) Thèse de Doctorat ès-sciences de l'Université de Nancy. 1932. 
nous que les chimistes, embarrassés dans le choix d'une méthode rationnelle, s'en tiennent généralement aux indications fournies par l'extrait sec dégraissé.

Désireux d'apporter quelques améliorations à cet état de choses, nous allons, dans le présent travail, passer en revue les principales constantes utilisées dans la détermination du mouillage du lait. Nous essayerons d'établir un choix judicieux basé sur de nombreuses expériences comparatives, réalisées dans les laboratoires de l'Ecole de Laiterie de l'Université de Nancy et à l'Etablissement fédéral d'Industrie laitière et de Bactériologie du Liebefeld à Berne.

Nous profitons de l'occasion pour remercier chaleureusement le Prof. M. Bouin, de Nancy, d'avoir bien voulu diriger nos recherches en les faisant bénéficier de sa grande expérience.

Nos remerciements s'adressent aussi au $D^{r}$ Koestren, chef du Iaboratoire chimique à l'Etablissement fédéral d'Industrie laitière au Liebefeld, qui ne nous a pas ménagé son précieux appui.

Les conseils expérimentés de ces deux savants ont grandement facilité l'accomplissement de notre tâche.

\section{Classification des constantes devant servir à la détermination du mouillage.}

Nous divisons notre travail en deux parties :
A. Les constantes physiques.
B. Les constantes chimiques.

A. Constantes physiques :

I. Cryoscopie.

II. Réfractométrie.

III. Résistivité électrique.

IV. Tension superficielle.

V. Viscosité.

B. Constantes chimiques :

I. Résidu sec dégraissé.

II. Extrait délactosé d'Ackermann.

III. Constante de Cornalba.

IV. Constante de Bordas et Touplain.

V. Constante moléculaire simplifiée réelle.

VI. Constante de Bouin.

Ire PARTIE.

\section{A. CONSTANTES PHYSIQUES.}

\section{LA CR YOSCOPIE.}

Définition. - La cryoscopie est la détermination de la température du point de congélation d'un liquide, au moment de l'apparition des premiers cristaux de glace. Cette température est soumise à des 
variations dues à la pression atmosphérique, mais les écarts enregistrés sont si faibles qu'ils ne nous intéressent point ici.

Le mouillage du lait élève la constante cryoscopique proportionnellement à la quantité d'eau additionnée.

Historique. - Winter démontrait en 1895, puis en 1897, que le point de congélation du lait était le même que celui du sérum du sang, par conséquent, que le lait et le sang avaient même concentration moléculaire. Il le fixe à $\Delta=-0^{\circ} 555$.

Par la suite, de très nombreux auteurs ont étudié cette constante au point de vue de la recherche du mouillage. Nous ne nous y arrêterons pas. Nous nous contentons de citer leur nom dans la bibliographie.

CAUSES dES VARIATIONS DU POINT cRYOSCOPIQUE. - a) Addition de matières solubles ; $b$ ) Acidité ; $c$ ) Fraude par mouillage ; d) Lait pathologique.

a) Addition de matières solubles. - L'addition au lait de solutions isotoniques augmente l'abaissement du point cryoscopique (lactose, biborate de soude, chlorure de sodium, bicarbonate de soude, bichromate de potasse, etc.).

En France, suivant l'arrêté du $1^{\text {er }}$ août 1907, tous les prélèvements officiels de lait sont additionnés de bichromate de potasse, en vue de leur conservation. Il convient done d'en tenir compte dans la cryoscopie. Il est nécessaire de faire une correction au $\Delta$, sachant que $1 \mathrm{gr}$. de ce sel augmente l'abaissement de la température de congélation de $-0^{\circ} 018$.

Cette correction enlève au procédé sa principale qualité, c'està-dire sa précision. Elle compljque aussi l'opération; e'est pour ces raisons que la détermination du point cryoscopique n'est pas employée dans les laboratoires de la répression des fraudes.

En outre, la facilité avec laquelle il est possible de masquer un mouillage par l'addition de substances solubles, le chlorure de sodium par exemple, rend la méthode imparfaite.

b) Acidité. - Un lait aura une température de congélation d'autant plus basse que son degré d'acidité sera plus fort. Ce phénomène s'explique par le fait d'un accroissement du nombre des molécules dissoutes à la suite de la transformation du lactose en acide lactique. Suivant la formule, 1 molécule de lactose hydraté donne 4 molécules d'acide lactique :

$$
\mathrm{C}^{12} \mathrm{H}^{22} \mathrm{O}^{11}+\mathrm{H}^{2} \mathrm{O}=4 \mathrm{C}^{3} \mathrm{H}^{6} \mathrm{O}^{3}
$$

Tous ceux qui se sont appliqués à des recherches sur la cryoscopie ont remarqué que le $\Delta$ d'un lait subissait des variations en rapport avec le nombre d'heures écoulées après la traite.

Stowckin [7] nous donne la formule par laquelle il convient de 
corriger le $\Delta$ dans les laits acides. Pour cela, il admet une acidité normale de $1 \mathrm{gr} .2$ d'acide lactique, qui n'intervient pas dans la correction, cette quantité représentant la teneur moyenne des laits immédiatement après la traite. D'après cet auteur, il convient de retrancher 0,05 du $\Delta$ trouvé, par gramme d'acidité au-dessus de $1 \mathrm{gr}, 20$. Il donne une formule globale permettant à la fois la correction due à l'acidité lactique, sans tenir compte de celle due à l'addition de bichromate de potasse, dans les cas de lait bichromaté.

$\mathrm{L} \times 0,05$ à retrancher du $\Delta$ trouvé.

$\mathrm{L}=\mathrm{A}-(1,20+\mathrm{Cr} \times 0,61)$.

$\mathrm{L}=$ acidité lactique formée après la traite.

$\mathrm{A}=$ acidité totale du lait.

0,61 = augmentation de l'acidité par $1 \mathrm{gr}$, de $\mathrm{K}^{2} \mathrm{Cr}^{2} \mathrm{O}^{7}$.

$1,20=$ acidité immédiate après la traite.

Cette correction n'est nullement négligeable pour des laits frais, à plus forte raison pour des laits vieux. D'autre part, la fermentation des laits est plus ou moins rapide selon la saison, la température du liquide, le nombre d'heures écoulées après la traite, le traitement du lait, la propreté des récipients, etc.

La cryoscopie doit donc être accompagnée du dosage de l'acidité. Ajoutons-y, le cas échéant, celui du dosage du bichromate. Nous voyons donc que la cryoscopie n'est plus une méthode de recherche du mouillage aussi rapide qu'on a voulu le prétendre.

Enfin, la détermination du $\Delta$ est rendue impossible dans les laits bichromatés en voie d'altération.

L'évaporation de l'eau du lait, à l'air libre, abaisse également le point de congélation. Il n'en est plus de même pour les laits stérilisés en vase clos.

c) Fraude par mouillage. - La dilution des éléments solubles du lait ayant pour effet de diminuer la concentration moléculaire retentit, 'de toute évidence, sur le point de congélation. La quantité d'eau ajoutée est directement proportionnelle à l'élévation vers le zéro du point cryoscopique; autrement dit, le mouillage rapproche de $0^{\circ}$ le $\Delta$ proportionnellement à la quantité d'eau pure ajoutée. Voici la formule de Winter qui permet d'apprécier ce mouillage :

$$
\mathrm{E}=\mathrm{V} \frac{\mathrm{a}-\Delta}{\mathrm{a}}
$$

$\mathrm{E}$ est le volume de l'eau contenue frauduleusement dans le volume $\mathrm{V}$ examiné.

a est l'abaissement normal : - 05555 .

$\Delta$ est l'abaissement observé et corrigé (acidité et conservateur).

LA CRYOSCOPIE ET LES LAITS PATHOLOQIQUES.

Des quelques analyses que nous avons de la composition des laits 
pathologiques, nous déduisons que, en règle générale, le $\Delta$ n'est pas influencé par les laits pathologiques. C'est ce qui fait la grande valeur de la cryoscopie. Nous avons dit " en règle générale ", car, comme toute règle, celle-ci comporte des exceptions, assez rares il est vrai.

Parmentier [3] examine quelques échantillons: Un colostrum de vache mammitique lui donne $-0^{\circ} 56$ et, einq jours plus tard, $-0^{\circ} 86$. $\mathrm{Au}$ bout de vingt-cinq jours, un nouvel échantillon marque un abaissement cryoscopique normal. Ce lait n'avait pas l'aspect naturel ; il était jaune trouble et grumeleux. Parmentier signale d'autres cas avec $-0^{\circ} 60$ et à $-0^{\circ} 58$. Chez une vache tuberculeuse amaigrie il trouve un $\Delta$ de $-0^{\circ} 48$, qui, trois jours après, lui donne :

Traite du matin $\ldots \ldots \ldots \ldots \ldots \ldots . \quad-0^{\circ} 54$
Traite du soir $\ldots \ldots \ldots \ldots \ldots \ldots .0^{\circ} 55$

Voici ses conclusions : " Le point de congélation cesse done d'être constant, normal, dans le cas d'inflammation locale, de mammite, et même, sans doute plus exceptionnellement, dans la tuberculose et dans les maladies graves de la vache."

D'après BARBATIS [8] :

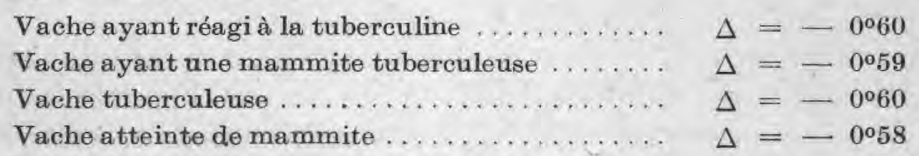

SchNorf [9] indique, dans plusieurs cas de mammite streptococcique, les points de congélation suivants :

$$
-0^{\circ} 565 ;-0^{\circ} 600 \text {; }-0 \circ 810
$$

Monvorsin [10], dans un cas de mammite tuberculeuse : $-0^{\circ} 510$; l'échantillon était totalement dépourvu de lactose.

VAN DeR LAan démontre que le sérum du sang et celui du lait oscillent parallèlement :

\begin{tabular}{|c|c|c|}
\hline & $\Delta$ sang & $\Delta$ lait \\
\hline Vache à pyélonéphrite & $-0^{\circ} 546$ & $-0^{0} 547$ \\
\hline Tuberculose des ganglions du grasset & $-0^{\circ} 541$ & $-0^{\circ} 539$ \\
\hline Tuberculose pulmonaire . & $-0^{\circ} 532$ & 一 $0^{\circ} 532$ \\
\hline Fracture du coxal & $-0^{\circ} 554$ & $-0^{\circ} 559$ \\
\hline Forte hémorragie $\ldots \ldots \ldots \ldots$ & $-0^{\circ} 532$ & $\longrightarrow 0^{\circ} 533$ \\
\hline
\end{tabular}

A ces chiffres, nous pourrions en ajouter d'autres, mais nous ne le jugeons pas nécessaire. En règle générale, les laits pathologiques diminuent ou augmentent le point de congélation du lait dans des proportions relativement faibles.

Valeur pratique de la cryoscopie. - Nous considérons la cryoscopie comme la constante la plus sûre, la plus exacte et la moins variable de toutes les constantes connues jusqu'à nos jours, pour autant qu'elle s'applique à des laits purs et vieux de quelques heures seulement. 
Malheureusement, cette constante comporte de nombreux inconvénients. C'est pourquoi elle ne sera jamais très répandue.

Nous avons cité, plus haut, les inconvénients dus : à l'addition de bichromate de $\mathrm{K}$; à l'acidité ; à la facilité de masquer un mouillage par addition de solutions isotoniques, etc.

Nous lui reprochons encore d'être d'une manipulation délicate exigeant un tour de main exercé.

Enfin, dans les cas de laits anormaux ou pathologiques, le $\Delta$ peut être en défaut; par conséquent, cette constante perd toute sécurité absolue.

D'après Parmentier [3], les écarts de cette constante sont grands : $-0^{\circ} 86$ à $-0^{\circ} 48$. Bien que ces chiffres représentent des exceptions, cela ne change pas les données du problème. Chaque fois que le chimiste se trouve en présence d'un point cryoscopique bas, il doit se demander s'il n'est pas en face d'une de ces exceptions. Dans aucun eas, il ne saurait affirmer un mouillage en basant uniquement ses observations sur le point de congélation.

Néanmoins, cette constante est précieuse, car elle apporte, en concordance avec d'autres chiffres, la preuve quasi irréfutable de la nature du lait.

La cryoscopie est employée en Allemagne et en Suisse, où les prélèvements officiels de lait se font d'une façon autre qu'en France. Les Américains, qui conservent par le froid les échantillons d'analyses, ont fait de la cryoscopie une méthode officielle d'analyse du lait. L'introduction de la cryoscopie en France serait désirable. Nous sommes certains qu'elle y rendrait des services appréciables. Pour cela il serait nécessaire de modifièr le système actuel de prélèvement des échantillons. Aujourd'hui, on se contente d'autres constantes, plus simples, plus rapides, plus nettes, mais qui offrent peut-être moins de garanties.

\section{LA RÉFRACTOMÉTRIE.}

La déviation que produit un rayon lumineux, passant obliquement d'un milieu réfringent dans un autre milieu plus réfringent, peut être mesurée par le réfractomètre.

Il existe de nombreux appareils pour opérer cette détermination. Les uns s'appliquent aux laits entiers (réfractomètre de Pulfrich, d'Abbe); d'autres nécessitent le recours à un milieu transparent et, pour eela, exigent la préparation du lacto-sérum. Seuls ces derniers nous intéressent, car le pouvoir réfringent du lait complet varie dans des limites trop larges pour permettre de servir à la détermination du mouillage. Nous ne nous arrêterons donc pas à étudier les trop nombreux modèles actuellement en usage. Nous citerons simplement, pour mémoire, celui qui nous paraît le plus pratique : le « réfractomètre à immersion " de Zeiss. Il permet de faire des essais en série avec une grande rapidité. 
Historique. - La détermination du pouvoir réfringent du lait a fait l'objet de nombreuses études. Nous ne les mentionnerons pas ici.

Nous renvoyons les lecteurs qui s'y intéressent aux travaux originaux ou aux ouvrages de chimie laitière mentionnés dans notre bibliographie.

Préparation du lacto-sérum. - C'est de cette opération que dépend la valeur réelle de la réfractométrie. La préparation du lactosérum peut se faire par de nombreuses méthodes, qui sont :

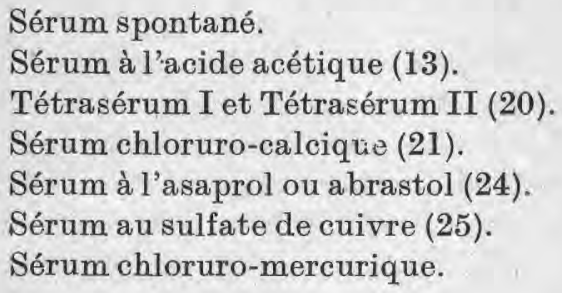

\section{CHOIX D'UNE MÉTHODE POUR LA PRÉPARATION DU SÉRUM.}

Des méthodes que nous avons vues précédemment, deux seulement méritent de retenir notre attention. Ce sont :

1. La méthode d'Ackermann.

2. La méthode d'Ambühl et Weiss.

Elles ont, toutes deux, l'avantage d'être rapides et de donner, dans le maximum des cas, des sérums clairs et dépourvus d'albumine: Cependant, la méthode d'Ackermann, aujourd'hui très étudiée, très répandue, semble réunir le plus grand nombre d'avantages pratiques ; c'est pourquoi nous lui donnons la préférence. En effet, dans la plupart des eas, la méthode' d'Ackermann permet de se passer de filtration. Ce n'est jamais le cas avec la méthode au chlorure mercurique, à laquelle on reproche également sa toxicité et son prix trop élevé. Mais nous verrons que la méthode d'Ackermann a aussi ses inconvénients.

Nous déconseillons l'emploi des autres procédés énumérés cidessus en raison des nombreux inconvénients qu'ils présentent et qui nuisent beaucoup à la valeur pratique de la réfractométrie. Les reproches les plus justifiés qu'on fait à ces méthodes sont :

1. Présence d'albumine dans le lacto-sérum susceptible d'augmenter l'indice de réfraction de $0^{\circ} 7$ à $3^{\circ}$ (réfractomètre de Zeiss).

2. Filtration lente, filtrat trouble.

3. Manque de sensibilité dans l'appréciation du mouillage par suite d'une trop forte dilution du lait par le réactif.

Les sérums préparés selon Ackermann ou selon Ambühl et Weiss 
présentent des avantages tels que nous ne nous occuperons que de ces deux procédés dans la détermination de l'indice de réfraction et de sa valeur " constante".

\section{SÉRUM CHLORURO-CALCIQUE.}

En 1901 déjà, Braun [15] utilisait le chlorure de ealcium pour la préparation du lacto-sérum. En 1907, le Dr Ed. Ackermann [21] reprend le chlorure de calcium comme coagulant. Il nous indique sa méthode qui lui donne un sérum dépourvu d'albumine, obtenu par simple décantation en faisant bouillir le lait avec une solution de $\mathrm{CaCl}^{2}$.

Ce réactif est obtenu de la façon suivante : L'auteur dissout 200 gr. de chlorure de calcium fondu dans la Q. S. d'eau distillée pour faire $1.000 \mathrm{em}^{3}$. Le poids spécifique de sa solution doit être de 1,1375 à $15^{\circ} \mathrm{C}$., et diluée au $1 / 10$, elle doit avoir un indice de réfraction au réfractomètre de Zeiss (R. Z.) de $26^{\circ}$.

On opère en mélangeant intimement, dans un tube muni d'un réfrigérant à reflux, $30 \mathrm{~cm}^{3}$ de lait avec $0 \mathrm{~cm}^{3} 25$ de ce réactif. On plonge ce mélange dans un bain-marie bouillant, pendant 15 minutes. Pour obtenir l'indice du pouvoir réfringent du lait à examiner, il suffit de décanter le sérum.

L'auteur a expérimenté sa méthode sur 2.800 essais, et il indique ses limites qui sont de 38,5 à 40,5 (R. Z.) ( $\mathrm{n}=1,3422$ et 1,3429).

De nombreux auteurs étudièrent encore cette méthode, chaudement recommandée par UTz [16], par C. MaI et S. Rothenfüsser [22], qui l'expérimentèrent eux-mêmes sur 5.000 échantillons. Ils démontrèrent que les variations de la teneur en graisse de même que celle du résidu sec dégraissé n'ont aucune influence sur l'indice de réfraction. Ils conclurent que cette méthode était la plus favorable à la recherche du mouillage. Leur indice moyen oscille autour de 39,5.

Avantages de cette méthode. - Elle est simple et rapide, n'exige pas toujours la filtration; elle est avantageusement connue et appréciée, parce qu'elle a été étudiée et expérimentée à fond.

Inconvénients. - Le sérum peut être trouble avec des laits très acides, à même d'élever l'indice R. Z. de $0^{\circ} 7$ à $2^{\circ}$ (n de 0,0003 à 0,0008 ), et ainsi dissimuler un léger mouillage.

C'est pourquoi H. LüHRIG et L. Kressner [27] estiment que la réfractométrie n'est pas applicable aux laits vieux, acides ou coagulés et que cette méthode doit se limiter anx laits frais.

PANCHAUD [28] dose pendant 25 jours un sérum chloruro-calcique et un sérum chloruro-mercurique d'un même lait ; il dose également l'acidité, puis les substances azotées du début et de la fin de l'expérience. 
Voici ses résultats :

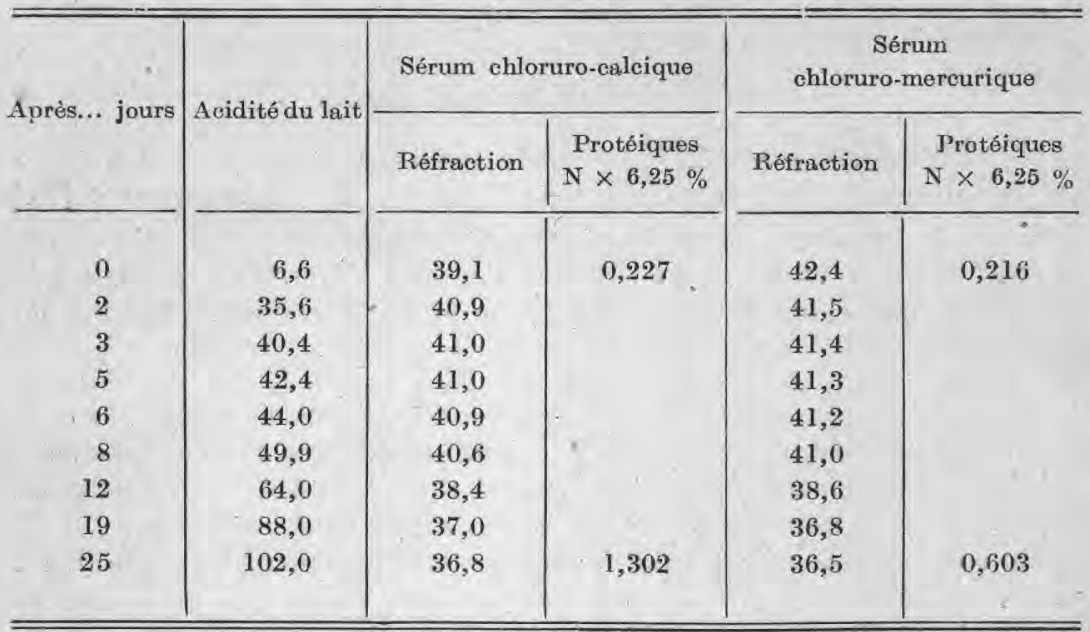

L'auteur en déduit : "Sérum chloruro-calcique. - A partir d'un certain chiffre d'acidité du lait $\left(40^{\circ}\right)$, la réfraction reste stationnaire, puis diminue régulièrement jusqu'à atteindre des valeurs inférieures à celles du début, alors que la teneur en azote est sensiblement augmentée. ")

"Sérum chloruro-mercurique. - Dès le début, la réfraction diminue lentement et régulièrement, alors même que la teneur en azote du sérum augmente moins fortement que dans le sérum chlorurocalcique. "

Panchadd explique que l'augmentation de l'indice du sérum chloruro-calcique " est due principalement à la formation de lactate de chaux aux dépens de la chaux de la caséine. Le lactate de chaux reste en solution dans le sérum, en élève la réfraction jusqu'à ce que toute la chaux soit saturée. Ce point atteint, la réfraction baisse, reprend la valeur qu'elle avait primitivement pour le lait frais, puis diminue encore. ")

Avec certains laits pathologiques, provenant spécialement d'animaux atteints d'affections mammaires, le sérum est trouble, les albuminoïdes n'étant pas précipités complètement. Rappelons, à ce sujet, les travaux de Metzger, Fuchs et Jesser [23], qui emploient $0 \mathrm{~cm}^{3} 5$ de la solution et font subir à l'indice trouvé une eorrection négative de $0^{\circ} 5$ au R. Z. (de 0,0002 sur n) à $17^{\circ} 5$ de température.

Mais dans tous les cas où il se produit un trouble avec la dose normale de $\mathrm{CaCl}^{2}$, on peut considérer ce signe comme une preuve d'anomalie du lait. 
MaI et RothenfüsSer [22] préconisent l'emploi du formol pour empêcher le développement de l'acidité.

ScHÜTZ et WEIN [29] appellent l'attention sur l'élévation de l'indice de réfraction au $\mathrm{CaCl}^{2}$ lorsque ce dernier reste quelque temps en contact avec le coagulum par suite d'une redissolution des sels calciques précipités à l'ébullition. L'examen doit avoir lieu dans la $1 / 2$ heure qui suit l'ébullition. Ces auteurs reconnaissent également que le formol empêche cette élévation pendant 12 jours.

Méthode d'Ambühl et Weiss. - Ceux-ci emploient le chloruremercurique pour obtenir à froid le lacto-sérum, qui doit servir à la réfraction:

Préparation de réactif. - Chauffer légèrement $125 \mathrm{gr}$. de bichlorure de mercure, chimiquement pur, avec de petites quantités d'HCl concentré $(d=1,184)$, que l'on ajoute jusqu'à dissolution. Après refroidissement, on complète à $100 \mathrm{~cm}^{3}$ avec $\mathrm{HCl}$ concentré. $30 \mathrm{~cm}^{6}$ d'eau distillée plus $0 \mathrm{~cm}^{3} 3$ de réactif doivent avoir un indice R. Z. \&े $20^{\circ} \mathrm{C}$. de $20,5(\mathrm{n}=1,3351)$.

Préparation du ś́rum. - A $30 \mathrm{~cm}^{3}$ de lait, ajouter $0 \mathrm{~cm}^{3} 3$ du réactif chloruro-mercurique, agiter et laisser au repos pendant 1 heure avant la filtration.

Le sérum est toujours clair, mais l'indice de réfraction, contrairement à celui du sérum chloruro-calcique, diminue avee le degré d'acidité du lait. On reproche à cette méthode son réactif coûteux, toxique et caustique.

\section{LA RÉFRACTOMÉTRIE ET LES LAITS PATHOLOGIQUES.}

L'indice de réfraction perd toute valeur dans les cas de laits pathologiques. Bien qu'il y ait certains cas où cet indice conserve ses variations normales, on peut dire que chaque fois que l'on remarque une diminution sensible du lactose, le pouvoir réfringent est également en baisse. Nous avons pu le constater souvent, aussi bien dans nos analyses que dans celles publiées par d'autres auteurs. Cela se comprend aisément si l'on examine les résultats expérimentaux de A. LataIx [26]. Nous y voyons, en effet, le rôle prépondérant gue joue le lactose dans la détermination de l'indice de réfraction. D'après cet auteur, le lactose représente plus des $5 / 6$ des matières en solution dans le lacto-sérum et il augmente, dans les mêmes proportions, l'indice de réfraction de l'eau distillée. Les indices de réfraction de ces sérums se classent exactement suivant la teneur en lactose des laits.

Dans certains cas pathologiques, la forte diminution du lactose n'est pas compensée suffisamment par l'augmentation des chlorures, comme dans la cryoscopie ou la C. M. S. A ce sujet, voici quelques résultats :

I. - Chez une vache malade, nous avons prélevé du lait de chaque quartier du pis : 
Analyse du 20.III.30

Indice de

réfraction

R. Z. (Sérum Lactose gr.

Ambühl

et Weiss)

I. Quartier A. G. . . . 4 41,7

II. Quartier P. G. . . . 37,7

III. Quartier A. D. . . . . .

IV. Quartier P. D. . . . . .
41,9

42,5 $\%$ gr.

46,503

34,451

45,827

48,130
Analyse du 26.III.30.

R. Z. Lactose

$\begin{array}{ll}34,5 & 22,90 \\ 32,4 & 15,96 \\ 41,8 & 48,25 \\ 41,7 & 47,32\end{array}$

II. -8 jours après l'avortement d'une vache, nous avons prélevé du lait de chacun des quartiers du pis (essais par la méthode d'Ambühl et Weiss) :

Analyse du 26.II.30

$\mathrm{n}_{\mathrm{d}} \quad$ R. Z. Lactose

I. Quartier A. G. . . 1,34059

II. Quartier P. G. ... 1,3415

III. Quartier A. D. . . 1,3426

IV. Quartier P. D. ... 1,3418
$34,319,52 \mathrm{gr}$. $\%$ or.

R. Z. Lactose $36,9 \cdot 35,23 \mathrm{gr} . \% \mathrm{oogr}$. $36,7 \quad 30,10$ gr. $\%$ or. $39,2 \quad 42,72$ gr. $\%$ o gr. $39,8 \quad 39,79 \mathrm{gr}$ \% \% gr. $41,0 \quad 47,52 \mathrm{gr}$ \% \% gr. $37,532,81 \mathrm{gr}, \%$ or. $39,1 \quad 44,39 \mathrm{gr} . \%$ gr.

A ces analyses nous pouvons ajouter encore celles de Monvorsin [10], de vaches à mammite tuberculeuse :

\section{Indice de réfraction}

$\mathrm{n}_{\mathrm{d}}$ R. Z

26.II.06. Quartiers sains ... 1,3428

26.II.06. Quartier atteint . $\quad 1,3382$

5.III.06. Quartier atteint. . . $\quad 1,3382$

6.III.06. Quartiers sains ... 1,3426

6.III.06. Quartier atteint. . . 1,3376

8.III.06. Quartier atteint... 1,3374

10.III.06. Quartier atteint.. $\quad 1,3375$ $\left(40^{\circ}\right)$

\section{Lactose}

$38,7 \mathrm{gr} . \%$ gr.

7,7 gr. $\%$ gr.

$4,8 \mathrm{gr} . \%$ gr.

$38,2 \mathrm{gr}$. $\%$ o gr.

$3,5 \mathrm{gr} . \% \mathrm{gr}$.

$4,2 \mathrm{gr} . \% \mathrm{or}$.

$2,9 \mathrm{gr} . \% \mathrm{gr}$.

RIPPERT [19] a fait observer qu'un indice de réfraction bas indiquait soit un mouillage, soit un lait pathologique. Il opérait avec des lacto-sérums préparés à l'acide acétique et préconisait l'emploi de la réfraction pour différencier un lait pathologique d'un lait sain. Sa méthode fut très discutée, puis réfutée.

Les résultats que nous publions plus haut n'ont pas pour but de montrer l'excellence de la méthode de Rippert. Au contraire, nous nous rangeons, et pour cause, à l'avis de ses contradicteurs. Souvent, en effet, des laits pathologiques préparés par la méthode acétique nous ont donné des indices de réfraction sensiblement au-dessus des limites maximales normales. Cela peut très bien s'expliquer par le fait de la présence d'albumine acéto-soluble dans le sérum, car, comme nous le dirons plus loin au sujet de la C. M. S., les laits pathologiques sont le plus souvent riches en albumine.

La préparation du sérum, libre d'albumine, comme c'est le cas dans nosessais, ne subit pas cette influence, et c'est la raison pour laquelle l'indice de réfraction suit la même courbe que celle de la teneur en sucre. 


\section{LA REFRACTION ET LES LAITS NORMAUX.}

Nous venons de voir que, dans les laits pathologiques, l'indice de réfraction varie avec la teneủr en lactose des lacto-sérums. Dans les cas de laits normaux, c'est-à-dire provenant d'animaux sains, le même phénomène se produit. Ainsi la détermination de l'indice de réfraction dépend de la teneur en lactose et en suit, en quelque sorte, les variations. La réfractométrie subira donc des variations au cours de la lactation, alors qu'elle restera sensiblement constante dans les laits de mélange.

La réfractométrie est un excellent moyen, surtout un moyen rapide, pour la détermination du mouillage. Mais en aucun cas, elle ne permet d'affirmer la fraude à elle seule. Elle est indépendante de l'écrémage du lait, de l'alimentation de l'animal, etc. Il n'est pas certain que l'indice soit influencé par le milieu et la race. On peut regretter qu'un aussi grand nombre de procédés pour la préparation du lacto-sérum soit encore actuellement en usage.

En règle générale, on peut admettre pour l'indice de réfraction les limites minima suivantes:

$38^{\circ}$ R. Z. pour la méthode chloruro-calcique.

$39^{\circ} \mathrm{R}$. Z. pour les méthodes acétiques.

$40^{\circ} \mathrm{R}$. Z. pour la méthode chloruro-mercurique.

Nous ne contesterons pas que des laits individuels, sains et purs, puissent avoir un chiffre inférieur à ceux qui sont indiqués ci-dessus. Ces cas sont néanmoins très rares, mais la prudence nous oblige de compter avec eux.

\section{VALEUR DE LA REFFACTOMÉTRIE POUR LA DETERMINATION DU MOUILLAGE.}

1. Lait de mélange. - La réfraction n'a de valeur absolue que sur les laits frais de grand mélange; dans ces cas seulement, elle peut se suffire à elle-même pour l'affirmation du mouillage. (Nous sous-entendons ici l'emploi des méthodes que nous avons recommandées.) L'indice de réfraction des lacto-sérums provenant de lait de mélange aura une valeur supérieure à celle de l'E. D. (résidu sec dégraissé), lequel, nous le démontrerons plus loin, est sujet aux variations saisonnières.

Nous appelons " lait de grand mélange », le lait mélangé provenant de plusieurs centaines d'animaux.

2. Laits individuels. - Dans les cas de laits individuels, la plus grande prudence est de rigueur. L'indice de réfraction perd ici toute valeur absolue et, par conséquent, ne se suffit plus à lui-même.

Le pouvoir réfringent est susceptible de nous donner des indications de la plus haute importance. Sa place s'impose dans tous les bulletins d'analyse. 
La réfraction d'un lacto-sérum de lait individuel compris entre 38 et 42 (R. Z.) pour la méthode d'Ackermann ou 40 à 44 (R. Z.) pour la méthode d'Ambühl et Weiss, n'indique pas nécessairement que le lait soit pur. Nous savons, en effet, qu'un mouillage de $10 \%$ peut parfois passer inaperȩu.

$\mathrm{Si}$, au contraire, le lait soumis à la détermination de l'indice de réfraction donne des chiffres inférieurs à 38 pour la méthode au chlorure de calcium et à 40 pour la méthode chloruro-mercurique, le lait devient suspect et nous nous trouvons en face de trois éventualités :

1. Laits pathologiques.

2. Laits vieux et acides.

3. Laits fraudés.

Dans ces trois cas, l'analyse chimique s'impose et c'est à elle qu'il appartiendra de juger le cas.

En dehors des deux méthodes de préparation de sérums dont nous venons de parler, la réfractométrie n'a pour nous qu'une valeur secondaire. En effet, pour ne parler que des méthodes à l'acide acétique, celles-ci sont parfois dans le cas de ne pas. révéler un mouillage de $15 \%$. De ce fait, nous admettons aisément qu'on puisse se passer de la réfraction dans un bulletin d'analyse.

L'emploi du réfractomètre à immersion de Zeiss donne la constante la plus rapidement obtenue que l'on connaisse pour le triage des laits, au point de vue de leur pureté.

La réfraction est actuellement très employée en Allemagne et en Suisse. Nous souhaitons qu'elle s'étende davantage encore, surtout en France, où elle est encore trop peu utilisée.

\section{LA DENSITÉ DU SERUM DU LAIT.}

Dietzsch [30] indique que la densité du sérum du lait, obtenu par coagulation spontanée, oscille entre 1,027 et 1,029. Il en propose l'emploi pour la détermination du mouillage. En 1884, il prépare son sérum au moyen de l'acide acétique comme coagulant. Cette méthode lui donne de bons résultats.

WIEGNER et JAKUWA [31] établissent deux formules qui, à l'aide de la constante R. Z., permettent de caleuler le poids spécifique du sérum, et auxquelles ils accordent la même valeur qu'à la réfraction pour la détermination du mouillage, et inversement.

Puis WIEGNER [32] nous donne des formules applicables au réfractomètre à immersion de Zeiss :

$$
\begin{aligned}
& \text { I. R. Z. }=970,88 \mathrm{~d}_{\frac{15}{15}}-957,06 \\
& \text { II. } d_{\frac{15}{15}}=0,00103 \text { R. Z. }+0,98578
\end{aligned}
$$


dans lesquelles $\mathbf{R}$. Z. est l'indice de réfraction avec le réfractomètre de Zeiss à $17^{\circ} 5 \mathrm{C}$., et $\mathrm{d}_{\frac{15}{15}}$, la densité du même sérum à $15^{\circ} \mathrm{C}$.

Ackermann $[21, d]$ contrôle la méthode des formules de Wiegner en opérant des dilutions jusqu'à $50 \%$. Il établit un diagramme dans lequel les résultats sont parfaitement concordants.

Les laboratoires ne possédant pas de réfractomètre pourront donc aisément connaître l'indice de réfraction des lacto-sérums à examiner au moyen de la prise de densité et de l'application des formules ci-dessus.

\section{LA RÉSISTIVITÉ ÉLETRIQUE DU LAIT.}

La rézistivité électrique du lait, ou "résistance spécifique ", est la résistance qu'oppose ce liquide au passage d'un courant électrique cornu, sur une longueur et une section connues. On la désigne généralement par $\rho$. La conductibilité est l'inverse de la résistivité. On la désigne par K.

Pour cette détermination, on emploie le pont de Wheatstone et la méthode de Kohlrausch [41].

L'eau pure oppose une grande résistance au courant électrique. 'Cette résistance diminue si l'on dissout dans l'eau une certaine quantité d'électrolytes, e'est-à-dire d'éléments capables de subir une dissociation hydrolytique. Cette résistance sera d'autant plus petite que la concentration en molécules dissociées sera plus grande.

Le lait, contenant dans son sérum des électrolytes, a une résistivité beaucoup plus petite que l'eau pure. Ainsi, l'addition an lait d'eau pure augmente la résistance spécifique de ce liquide proportionnellement à la quantité additionnée. Telle est l'origine de l'emploi de la "détermination de la conductibilité du lait" pour la détermination du mouillage.

Technique., - On ne peut faire usage du courant continu ; par électrolyse, il modifierait les propriétés du lait. L'opération nécessite l'emploi du courant alternatif, dont la mesure se fait par un téléphone ordinaire.

Les premiers essais relatifs à la conductibilité électrique du lait ont été faits par DoHrmann [33], qui opéra avec un galvanomètre et un pont de Wheatstone, à la température de $10^{\circ} \mathrm{C}$. La même année, ThöRner [34] opérait suivant la méthode de Kohlrausch, à la température de $17^{\circ} \mathrm{C}$. Ces deux auteurs donnèrent leur nom à cette méthode et indiquèrent que la résistance électrique du lait oscille de 180 à 200 ohms.

JoRDIs [35] indique une conductibilité moyenne de $\mathrm{K}=46,26 \times$ $10^{-4}$ et fait des essais en additionnant des électrolytes, tels que le biborate ou le bicarbonate de soude, lesquels élèvent rapidement cette constante. 
Lesage et Dongier [36] trouvent une constante variant de $\mathrm{K}=49,29 \times 10^{4}$ à $50,68 \times 10^{-4}$ et font remarquer que la résistivité électrique d'un lait, étant de $254 \mathrm{ohms}$ immédiatement après la traite, n'est plus que $202 \mathrm{ohms,} 48$ heures après la traitè.

Schnorf [37] fixe cette constante à $\mathrm{K}=48,50 \times 10^{-4}$ à $25^{\circ} \mathrm{C}$., après l'avoir expérimentée sur 3.730 échantillons. Ces écarts sont de $38,69 \times 10^{-4}$ à $62,99 \times 10^{-4}$.

Le $\mathrm{D}^{\mathrm{r}}$ Binagli [38] indique les chiffres suivants, établis sur 36 échantillons de lait pur :

Lait de brebis. 49,43 à 51,72 (moyenne $K_{25}=50,40 \times 10-4$ )

Lait dé chèvre 47,01 غे 49,96 (moyenne $\mathrm{K}_{25}=49,00 \times 10-4$ )

Lait de vache : 47,97 à 49,78 (moyenne $K_{w 5}=48,70 \times 10-4$ )

STROHECKER [39] indique une résistivité spécifique du lait normal variant entre $44,00 \times 10^{-4}$ et $54,00 \times 10^{-4}$.

Il ajoute que les valeurs inférieures à ces ehiffres indiquent l'addition d'eau, tandis que les valeurs supérieures dénoncent un trouble dans la sécrétion.

\section{VALEUR PRATIQUE dE LA RÉSISTIVITÉ ÉLECTRIQUE DU LAIT.}

L'idée de se servir de cette méthode pour la détermination du mouillage du lait est théoriquement excellente. Nous disons " théoriquement ", parce que l'addition d'eau pure abaisse régulièrement cette constante; la matière grasse, essentiellement variable dans le lait, ne lui fait subir aucune variation. D'autre part, sur un même lait, la résistivité électrique reste constante chez un même animal, pour autant que celui-ci reste dans des conditions normales de lactation. L'addition de petit-lait ou l'écrémage ne masque pas le mouillage. De même, ce procédé révèle spontanément la présence de conservateurs, tels que le carbonate ou le biborate de soude, l'acide borique, etc., additionnés frauduleusement an lait. A cela, nous pourrions encore ajouter la simplicité de l'opération et sa rapidité. Néanmoins, leśs inconvénients sont plus nombreux que les avantages. Le premier inconvénient est dû aux écarts par trop considérables que l'on peut rencontrer $\left(38,69 \times 10^{-4}\right.$ à $\left.62,99 \times 10^{-4}\right)$, de sorte qu'il est impossible d'affirmer un mouillage de $40 \%$ sur un lait normal. Ainsi le mot "constante", appliqué trop généreusement à ce procédé, risque de perdre le sens exact qu'on lui attribue.

Un autre inconvénient de ce procédé, c'est que la fraude se fait ordinairement avee de l'eau contenant une quantité plus ou moins forte d'électrolytes, et non avec de l'eau distillée, de sorte qu'il est impossible de connaître l'augmentation de la résistivité correspondant à un mouillage déterminé. TILLMANn et OBERMEIER [40] font remarquer à ce sujet que l'eau en question peut avoir une conduetibilité 
égale à celle du lait et ainsi laisser au mélange une conductibilité normale.

Enfin, la résistivité du lait est influencée par la fermentation lactique. L'acide lactique dissout une petite quantité de phosphate tricalcique, lequel augmente la concentration en électrolytes du lait et diminue ainsi sa résistance spécifique.

De même, le caséinate de chaux dissous par la matière protéique influence la conductibilité. Ch. Porcher et A. Chevaldier [55] préparènt des solutions de caséinate de chaux, contenant de la caséine et de la chaux en quantité variable, mais ayant la même résistivité électrique. Ces auteurs dissolvent une même quantité de chlorure de sodium dans chacune de ces solutions et trouvent alors des résistances électriques différentes.

Pour ces raisons, la résistivité électrique ne compte pas beaucoup d'adeptes et l'on peut dire aujourd'hui que ce procédé n'est plus employé pour la détermination du mouillage. La résistivité électrique a trouvé d'autres emplois, spécialement dans la recherche des laits pathologiques, où nous voyons cette constante s'élever très rapidement. (Schnorf [37], dans un cas l'a treuvée égale à 125,9 $\times 10^{-4}$.) Ce fait s'explique par l'augmentation d'électrolytes, spécialement le chlorure de sodium. Cette détermination permet de découvrir à temps une perturbation dans la sécrétion lactée et aussi le début des maladies de la mamelle.

\section{LA TENSION SUPERFICIELLE DU LAIT.}

On la mesure au moyen de l'ascension du liquide dans un tube capillaire ou par la numération des gouttes de $5 \mathrm{~cm}^{3}$ de lait, au moyen d'un compte-gouttes normal qui débite 100 gouttes d'eau distillée pour $5 \mathrm{~cm}^{3}$ à $15^{\circ} \mathrm{C}$.

La tension superficielle du lait de vache est inférieure à celle de l'eau. Cette diminution est due aux matières en suspension dans le lait. La tension superficielle augmente dans le lacto-sérum.

La tension superficielle de l'eau à $20^{\circ}$ est de 7,390 . A la même température, le lait a une tension superficielle qui varie de 5,060 à 5,726 , d'après KOBLER [42].

Le colostrum a une tension superficielle moindre, par suite de sa richesse en substances azotées. Cette tension s'accroît dans la masse lorsque la composition du colostrum se rapproche de celle d'un lait normal.

Les laits pathologiques montrent de grandes variations dans leur tension superficielle.

Burri et Nussbaumer [43] remarquent qu'en laissant le lait au repos pendant 12 heures, à la température ordinaire, la tension superficielle s'abaisse jusqu'à une certaine limite, où elle se stabilise. Cette 
limite est atteinte d'autant plus rapidement que la température est plus basse. En réchauffant le lait, on n'obtient pas une élévation de la tension superficielle. Cette dernière semble être fixée par le refroidissement du lait. La raison de ce phénomène n'est pas due au fait de la solidification des globules gras à basse température, puisque ceux-ci reprennent toute leur fluidité lorsque la température s'élève. Il est possible que ce phénomène résulte d'un changement irréversible de la easéine.

Nous savons que la tension superficielle dêpend des matières en suspension du lait, et que celles-ci peuvent varier dans de grandes proportions. Conséquemment, cette constante varie avec ces matières, ce qui la rend impropre à la recherche des fraudes.

\section{LA VISCOSITE DU LAIT.}

La viseosité du lait, ou frottement intérieur, se mesure en prenant comme unité la viscosité de l'eau, à une même température et à une même pression. La mobilité est le contraire de la viscosité. Cette dernière dépend des matières en suspension et des colloïdes. La caséine et la graisse sont les deux principaux facteurs susceptibles d'influencer la viscosité. La mobilité de la caséine augmente ou diminue suivant les variations de la température. La graisse est plus compacte à une température basse qu'à une température élevée, ce qui démontre que la viscosité diminue avee la température. Oertel [44] dit que le lactose n'a pas grande influence sur la viscosité. Par contre, il constate que la vitesse d'écoulement dépend : $1^{0}$ de la caséine ; $2^{\circ}$ de la graisse, et $3^{\circ}$ de l'albumine. Il fait, sur un même lait, l'expérience suivante :

\begin{tabular}{cccc}
\multicolumn{4}{c}{ Viscosité de : } \\
Lait entier & Petit-lait & Petit-lait & Petit-lait \\
162,6 & 147,3 & sans caséine & sans caséine et albumine \\
$162,116,2$ & 113,1
\end{tabular}

Oertel en déduit qu'il existe un rapport direct entre l'extrait sec: total et la viscosité.

Pour déterminer la viscosité du lait, on peut se servir du viscosimètre de Reischauer, en appliquant la formule suivante :

$$
\mathrm{X}=\frac{\mathrm{gw} \cdot \mathrm{S}^{2}}{\mathrm{gm}^{2}}
$$

$\mathrm{X}^{\circ}=$ viscosité $; \mathrm{gw}=$ poids de l'eau qui s'écoule pendant un temps. connu;

$\mathrm{S}^{2}=$ poids spécifique, $\mathrm{gm}=$ poids du lait écoulé pendant le même temps.

Cette détermination peut être faite plus simplement an moyen de l'appareil de Micault. Cet instrument a une contenance calculée de telle sorte qu'un volume d'eau distillée à $15^{\circ}$ de température s'écoule 
en 100 secondes. L'écoulement d'un même volume de lait se fait en un temps variable, de 160 à 200 secondes.

Nous avons dit plus haut que plus la température s'élève, plus la viscosité diminue. Nous citons, à l'appui, l'expérience de Soxhlet [46].

$\begin{array}{ccc}\text { Tempéràture } & \text { Eau distillée } & \text { Lait } \\ 0^{\circ} & 100 & 221,0 \\ +5^{\circ} & 100 & 207,7 \\ +10^{\circ} & 100 & 190,6 \\ +15^{\circ} & 100 & 188,7 \\ +20^{\circ} & 100 & 211,7 \\ +25^{\circ} & 100 & 175,9 \\ +30^{\circ} & 100 & 169,0\end{array}$

Le même phénomène se produit pour l'eau distillée, mais le lait a un frottement intérieur qui diminue plus rapidement que celui de l'eau, par l'élévation de la température.

\begin{tabular}{crrcrl} 
Température & \multicolumn{1}{c}{ Eau } & \multicolumn{1}{c}{ Lait } & Température & Eau & Lait \\
$0^{\circ}$ & 100,00 & 100,00 & $+20^{\circ}$ & 51,65 & 49,47 \\
$+5^{\circ}$ & 87,19 & 81,99 & $+25^{\circ}$ & 54,27 & 43,18 \\
$+10^{\circ}$ & 75,76 & 65,30 & $+30^{\circ}$ & 49,86 & 38,13 \\
$+15^{\circ}$ & 67,08 & 57,26 & & &
\end{tabular}

Koblen [42] observe que la viscosité reste constante pendant longtemps dans les laits provenant d'animaux sains. Il indique un coefficient de viscosité entre 1,60 et 2,0 (eau distillée $=1$ ).

Cavazoni [47] indique 167 à 203 pour le lait de vache (eau distillée $=100) ; 201$ à 215 pour lait de chèvre (eau distillée $=100$ ).

Lucrus [48] trouve dans les laits de mélange, pour la traite du matin, des variations de 171,6 à 178,9 et, pour les traites du soir; 179,3 à 184,9 (eau distillée $=100$ ).

Dans les cas de lait pathologique, la viscosité peut augmenter dans de très fortes proportions.

Le colostrum a une viscosité très élevée, en vertu de sa teneur en albumine. D'autre part, le frottement intérieur peut dépendre de la pullulation d'espèces microbiennes (lait visqueux, lait filant). Nous ne-voyons pas la possibilité d'employer le coefficient de viscosité des laits pour en déterminer le mouillage. Les limites extrêmes entre lesquelles un lait peut varier normalement sont trop grandes. 